\title{
Simulation of Second Harmonic Ultrasound Fields
}

Du, Yigang; Jensen, Henrik; Jensen, Jørgen Arendt

Published in:

Proceedings of IEEE International Ultrasonics Symposium

Link to article, DOI:

10.1109/ULTSYM.2010.0553

Publication date:

2010

Document Version

Early version, also known as pre-print

Link back to DTU Orbit

Citation (APA):

Du, Y., Jensen, H., \& Jensen, J. A. (2010). Simulation of Second Harmonic Ultrasound Fields. In Proceedings of IEEE International Ultrasonics Symposium (pp. 2191-2194). IEEE. https://doi.org/10.1109/ULTSYM.2010.0553

\section{General rights}

Copyright and moral rights for the publications made accessible in the public portal are retained by the authors and/or other copyright owners and it is a condition of accessing publications that users recognise and abide by the legal requirements associated with these rights.

- Users may download and print one copy of any publication from the public portal for the purpose of private study or research.

- You may not further distribute the material or use it for any profit-making activity or commercial gain

- You may freely distribute the URL identifying the publication in the public portal

If you believe that this document breaches copyright please contact us providing details, and we will remove access to the work immediately and investigate your claim. 


\title{
Simulation of Second Harmonic Ultrasound Fields
}

\author{
Yigang $\mathrm{Du}^{1,2}$, Henrik Jensen ${ }^{2}$ and Jørgen Arendt Jensen ${ }^{1}$ \\ 1) Center for Fast Ultrasound Imaging, Department of Electrical Engineering, Bldg. 349, \\ Technical University of Denmark, DK-2800 Kgs. Lyngby, Denmark. \\ 2) BK Medical Aps, Mileparken 34, DK-2730 Herlev, Denmark. \\ Corresponding email: yd@elektro.dtu.dk
}

\begin{abstract}
A non-linear ultrasound imaging simulation software should be capable of simulating the non-linear fields for any kind of transducer, focusing, apodization, and attenuation. At present, a major issue is the overlong simulation time of the non-linear software. An Angular Spectrum Approach (ASA) using a quasi-linear approximation for solving the Westervelt equation can simulate the second harmonic pressure at any distance. Therefore, it shortens the execution time compared with the operator splitting method. The purpose of this paper is to implement the monochromatic solution for the second harmonic component based on ASA and Field II, and to compare with results from the simulation program Abersim. A linear array transducer with a center frequency of $4 \mathrm{MHz}$ and 64 active elements is used as the transmitting source. The initial plane is $5 \mathrm{~mm}$ away from the transducer surface, and the fundamental pressure is calculated by Field II. The second harmonic pressure in $k$-space along the propagating direction is calculated as an auto-convolution of the fundamental pressure multiplied by an exponential propagating coefficient. In this case, the second harmonic pressure can be calculated using ASA for any plane parallel to the initial plane. In the focal plane (elevation-lateral) at $60 \mathrm{~mm}$ from the transducer surface, calculated by ASA, the RMS errors for the fundamental component are $2.66 \%$ referred to Field II and $4.28 \%$ referred to Abersim. For the second harmonic component, the RMS error is $0.91 \%$ referred to Abersim.
\end{abstract}

Keywords: angular spectrum approach, second harmonic pressure, Abersim, Field II

\section{INTRODUCTION}

Non-linear ultrasound fields can be simulated in many different ways. An operator splitting method (OSM) combined with Field II [1] [2] for simulating non-linear ultrasound fields has been presented in 2002 [3]. A current popular non-linear ultrasound simulation software is called Abersim [4] [5] [6] and developed at the Department of Circulation and Medical Imaging (ISB) at the Norwegian University of Science and Technology (NTNU). However, the long simulation time is an essential problem to nearly all types of non-linear softwares. For instance, Abersim [7] simulates a non-linear acoustic field based on OSM. Therefore, the acoustic propagation has to be simulated in incremental steps, and the pressure field of each simulated plane parallel to the transducer surface has to be calculated based on the previous plane. This will increase the execution time, if the simulated point is far away from the transducer, since the step size between two nearby planes should be small enough to satisfy the corresponding assumption of the Earnshaw or Poisson solution [8].
To avoid the long time calculations, an angular spectrum approach (ASA) [9] [10] is used to solve the non-linear Westervelt equation [11]. In this study, the acoustic field at any distance from the transducer surface can be calculated in one step based on an acoustic source plane and distance between source and the simulated plane. This makes it possible to simulate the non-linear fields for any kind of transducer, focusing, apodization, and attenuation. Field II is used to calculate the acoustic source plane, which is $5 \mathrm{~mm}$ away from the transducer surface, and it is assumed that non-linear effects are negligible at this distance. The second harmonic pressure is generated after propagation and calculated by the ASA based on the source plane. This is called Field-II-based ASA.

Four acoustic planes for calculating the second harmonic components including one plane at the focal distance from the transducer surface and three planes at three random depths are simulated in this paper. The results obtained from FieldII-based ASA are compared to the Abersim program using the same transducer with the same initialization. The computation time between Field-II-based ASA and Abersim is listed and discussed at the end of the paper.

\section{THEORY}

The non-linear Westervelt equation [12] is expressed by

$$
\left(\nabla^{2}-\frac{1}{c_{0}^{2}} \frac{\partial^{2}}{\partial t^{2}}\right) p=-\frac{\beta}{\rho_{0} c_{0}^{4}} \frac{\partial^{2} p^{2}}{\partial t^{2}},
$$

where $c_{0}, t$ and $\beta$ are sound speed, time and non-linear coefficient, respectively. It describes the propagation of nonlinear acoustic pressure $p$ in a lossless medium with the density of $\rho_{0}$. The analytical ASA solution is given by [13] [14]

$$
\begin{aligned}
\hat{P}_{2}\left(k_{x}, k_{y}, z\right)= & \frac{\beta k^{2}}{2 \pi^{2} \rho_{0} c_{0}^{2}} \iint \frac{\hat{P}_{1}\left(k_{x}^{\prime}, k_{y}^{\prime}, 0\right)}{k_{z 2}^{2}-\left(k_{z}^{\prime}+k_{z}^{\prime \prime}\right)^{2}} \\
& \times \hat{P}_{1}\left(k_{x}-k_{x}^{\prime}, k_{y}-k_{y}^{\prime}, 0\right) \\
& \times\left(e^{-j z\left(k_{z}^{\prime}+k_{z}^{\prime \prime}\right)}-e^{-j k_{z 2} z}\right) d k_{x}^{\prime} d k_{y}^{\prime} . \\
k_{z}^{\prime}=\sqrt{k^{2}-\left(k_{x}^{\prime}\right)^{2}-\left(k_{y}^{\prime}\right)^{2}} & \sqrt{k^{2}-\left(k_{x}-k_{x}^{\prime}\right)^{2}-\left(k_{y}-k_{y}^{\prime}\right)^{2}} \\
k_{z}^{\prime \prime}= & \sqrt{4 k^{2}-k_{x}^{2}-k_{y}^{2}}
\end{aligned}
$$

where $\hat{P}_{1}$ and $\hat{P}_{2}$ are the fundamental and second harmonic pressures in the Fourier domain, $k$ is the wave number, and $k_{x}, k_{y}, k_{z}$ are wave numbers along the corresponding axes. 


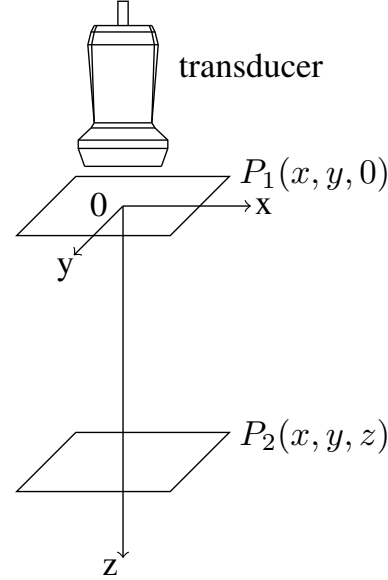

ASA

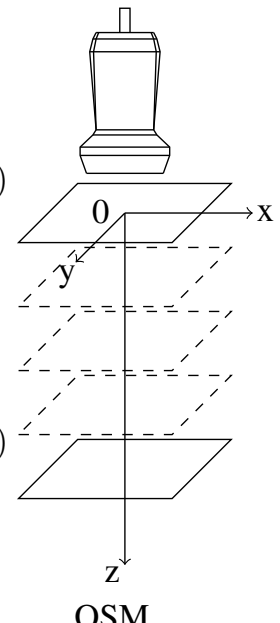

OSM
Fig. 1: Acoustic propagation simulation, ASA vs OSM. Planes $P_{1}$ and $P_{2}$ are parallel to the transducer surface. ASA: Angular Spectrum Approach. OSM: Operator Splitting Method. Coordinate: $x$-lateral, $y$-elevation, $z$-axial

A systematic view of the acoustic propagation is shown in Fig. 1. $P_{1}$ (solid plane on the top) is the linear ASA and OSM source calculated by Field II according to the types and settings of the transducers. The second harmonic pressure $P_{2}$ (solid plane on the bottom) is calculated through (2) by using the ASA as shown in the left graph in Fig. 1. Compared to ASA, OSM has to calculate a series of planes with a small step size until obtaining $P_{2}$ as shown in the right graph in Fig. 1. The specific theory for Field-II-based OSM can be found in [15] and using OSM for Abersim is described in [7].

\section{METhOD}

\section{A. Transducer settings}

A linear array transducer with 64 active elements is used as the emitting source. Its center frequency is $4 \mathrm{MHz}$, and each element has a $4.5 \mathrm{~mm}$ height and $0.208 \mathrm{~mm}$ width. The pitch is the same as the width for each element, since the kerf in Abersim is not implemented and is set to zero. Thus, the whole acoustic area is $4.5 \times 13.312 \mathrm{~mm}^{2}$. Both the elevation and lateral focal distances are $60 \mathrm{~mm}$ from the transducer surface.

\section{B. Field-II-based non-linear ASA}

The initial settings of the transducer are modeled by the function "xdc_focused_array" [16] in the Field II program. The ASA source plane is $5 \mathrm{~mm}$ from the transducer surface. It has $48 \times 192(y-x$ plane $)$ points with spatial sampling intervals of $d x=0.069333 \mathrm{~mm}$ and $d y=0.09375 \mathrm{~mm}$ which are less than half wavelength $(\lambda / 2=0.18529 \mathrm{~mm})$ according to Nyquist's Sampling Theorem. Therefore, the area of the plane is $4.5 \times 13.312 \mathrm{~mm}^{2}$ the same as that of the transducer surface. $P_{1}$ for each point on the source plane is calculated using the function "calc_hp" [16] of Field II. Then $\hat{P}_{1}\left(k_{x}^{\prime}, k_{y}^{\prime}, 0\right)$ in (2) is the 2D spatial Fourier transform of $P_{1}$. The second harmonic pressure $P_{2}$ is the 2D spatial inverse Fourier transform of $\hat{P}_{2}\left(k_{x}, k_{y}, z\right)$, which is calculated by (2) and implemented using Matlab.

\section{Abersim}

The exact same transducer is used in Abersim. The function "flags" in Abersim are $[1,1,1,0,0,0,3]$. The first three flags represent that diffraction, non-linearity, and attenuation are switched on. The next three flags are zeros, which denote homogeneous medium, non-annular transducer, and equidistant steps. The last flag is a history flag and " 3 " means that Abersim will store each pulse at each depth. The detailed specification for "flags" can be found on page 30-31 of [7]. Water is used as the material where the ultrasound propagates. The concept is that the acoustic pressure is calculated by Abersim in an attenuating medium to completely simulate the real world. The results are used as references and compared to Field-II-based ASA, which is the solution to the lossless Westervelt equation in this study, since the attenuation is fairly low in water. The sound speed in Abersim is $1482.3 \mathrm{~m} / \mathrm{s}$ by default, which is also used in the Field-II-based ASA simulation. The identical plane with the same number points and spatial intervals is used in the Abersim simulation. In OSM, a number of parallel planes should be generated in turn. The step size between two nearby planes is $0.5 \mathrm{~mm}$ set by Abersim based on the imaging frequency.

\section{RESUlts}

Fig. 2 illustrates the comparisons of the monochromatic pressure at $4 \mathrm{MHz}$ between the different methods for both the fundamental and second harmonic components along the lateral direction at $y=0$ on the left and along the elevation direction at $x=0$ on the right in the focal plane at $z=60 \mathrm{~mm}$ according to the coordinates of Fig. 1. The FWHMs (full width at half maximum) of the main lobe and the first sidelobe in $\mathrm{dB}$ for both the fundamental and second harmonic components using the different methods are shown in Table I. The entire simulated plane at the focal distance for the different methods is shown in Fig. 3 which also demonstrates the narrower beamform and better focus of the second harmonic component. The quantitative differences between the different methods are calculated as the RMS (root mean square) error by

$$
\text { RMS error }=\sqrt{\frac{\sum\left(P_{\mathrm{ASA}}(\vec{r})-P_{\mathrm{Ref}}(\vec{r})\right)^{2}}{\sum P_{\mathrm{Ref}}(\vec{r})^{2}}} \times 100 \%,
$$

where $P_{\mathrm{ASA}}(\vec{r})$ is the monochromatic pressure at one simulated plane calculated by Field-II-based ASA, and $P_{\operatorname{Ref}}(\vec{r})$ is the reference pressure at the same plane which may be calculated by Field II for the fundamental component or by Abersim for the fundamental or second harmonic components. The sum in the denominator overs the square of each difference between pressures calculated by Field-II-based ASA and references at each position of the simulated plane. Then it is divided by the sum of the square of the references. The square root of the quotient is called RMS error. For the fundamental component, the RMS errors are $2.66 \%$ compared to Field II and $4.28 \%$ compared to Abersim. For the second harmonic component, the RMS error is $0.91 \%$ compared to Abersim.

To further validate the Field-II-based ASA, planes at different distances and parallel to the transducer surface are calculated. Fig. 4 displays the comparisons of calculations at 

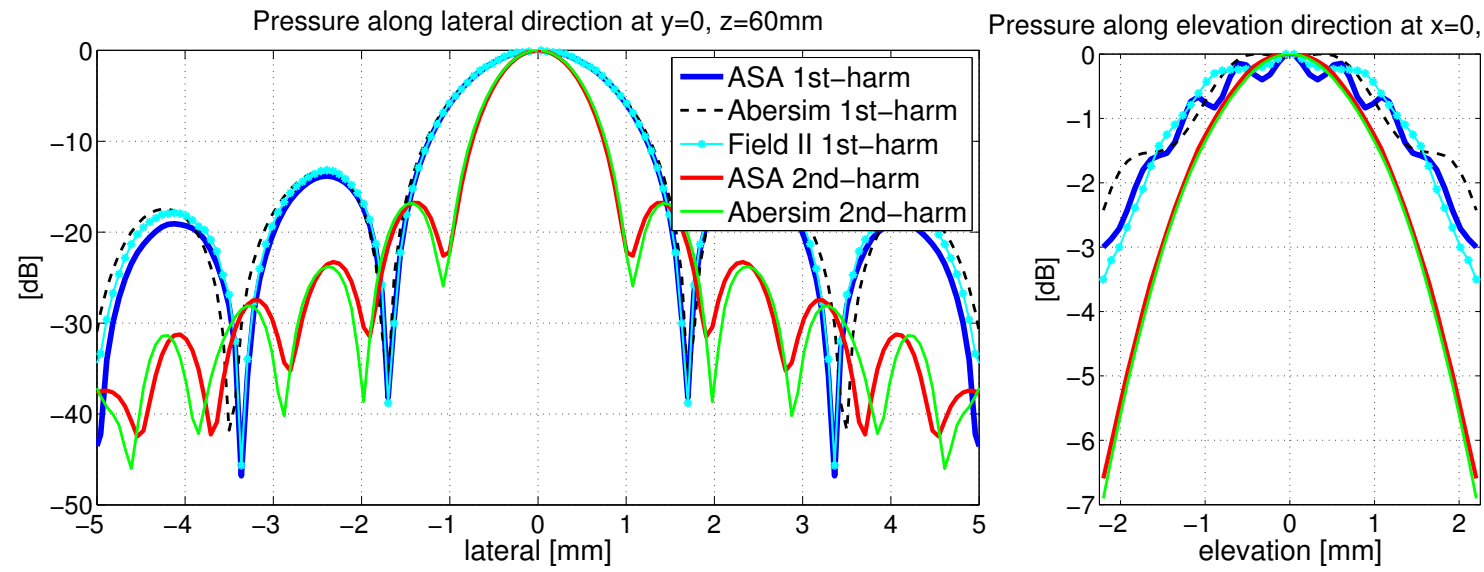

Fig. 2: Results for a linear array transducer with 64 active elements and $4 \mathrm{MHz}$ center frequency. Results are at the focal plane $60 \mathrm{~mm}$ from the transducer surface. 1st-harm = fundamental component and 2nd-harm = second harmonic component. The right figure has the same legend as the left.
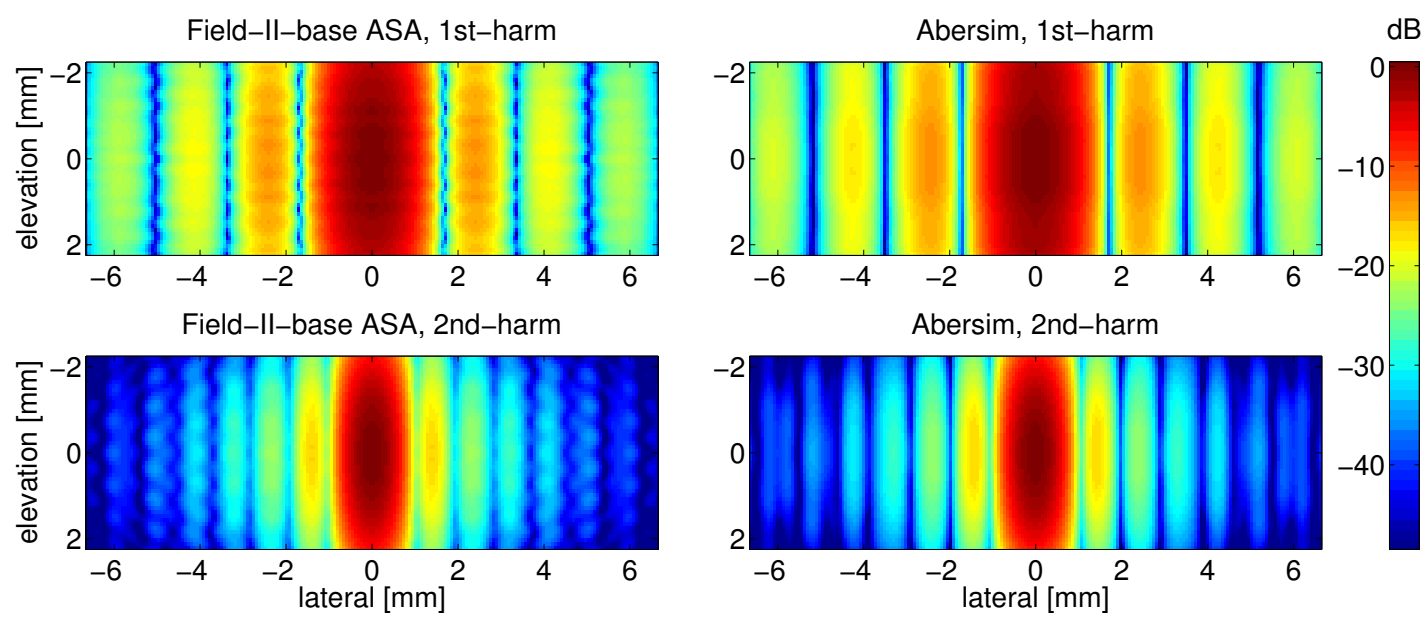

Fig. 3: Simulated plane at the focal distance, responses at $60 \mathrm{~mm}$ away from and parallel to the transducer surface

\begin{tabular}{lcc} 
& FWHMs of mainlobe & First sidelobe \\
\hline Fundamental component & & \\
Field-II-based ASA & $2.02 \mathrm{~mm}$ & $-13.82 \mathrm{~dB}$ \\
Abersim & $2.07 \mathrm{~mm}$ & $-13.28 \mathrm{~dB}$ \\
Field II & $2.04 \mathrm{~mm}$ & $-13.19 \mathrm{~dB}$ \\
\hline Second harmonic component & & \\
Field-II-based ASA & $1.21 \mathrm{~mm}$ & $-16.77 \mathrm{~dB}$ \\
Abersim & $1.24 \mathrm{~mm}$ & $-16.81 \mathrm{~dB}$ \\
\hline
\end{tabular}

TABLE I: Quantitative comparisons for different simulation methods

$y=0$ along the lateral direction at depths of $z=35 \mathrm{~mm}$, $z=45 \mathrm{~mm}$ and $z=70 \mathrm{~mm}$, respectively. The RMS errors are calculated between Field-II-based ASA and Abersim for the simulated planes with different distances from the transducer surface as shown in Table II.

\section{Computation time}

The computation time for the ASA mostly depends on the extent of zero-padding of the 2D spatial Fourier transform. To avoid circular convolution, the pressure $P_{1}$ should be zeropadded before applying the Fourier transform. It is a compromise, since the larger the zero-padding is the more accurate the

\begin{tabular}{c|ccc} 
& $\mathrm{z}=35 \mathrm{~mm}$ & $\mathrm{z}=45 \mathrm{~mm}$ & $\mathrm{z}=70 \mathrm{~mm}$ \\
\hline AS-Fd-1 & $2.03 \%$ & $2.39 \%$ & $2.10 \%$ \\
AS-Ab-1 & $5.63 \%$ & $2.68 \%$ & $3.37 \%$ \\
AS-Ab-2 & $3.75 \%$ & $1.13 \%$ & $0.83 \%$ \\
\hline AS-Fd-1: ASA compared to Field II for 1st-harm \\
AS-Ab-1: ASA compared to Abersim for 1st-harm \\
AS-Ab-2: ASA compared to Abersim for 2nd-harm
\end{tabular}

TABLE II: RMS errors for simulated planes with different distances from the transducer surface

ASA results and the longer the calculation time will be [14]. Table III displays the RMS errors and computation time for different methods and zero-padding numbers. The computer used to do the simulations has 4 cores Intel(R) Core $^{\mathrm{TM}_{2}}$ Quad $2.4 \mathrm{GHz}$ Q6600 CPU and $4 \mathrm{~GB}$ memory. The calculation is executed by Matlab 7.8.0 (R2009a) under the Microsoft Window XP Professional x64 Edition operating system. When zeropadding numbers are equal or larger than 6 , the simulation results for the two methods are fairly comparable, and the computation time of ASA is 2 hours, whereas Abersim needs 2 days to simulate the same data with the same computer. Furthermore, the Field-II-based ASA can be implemented and run on cluster machines. In this way, the simulation can be 

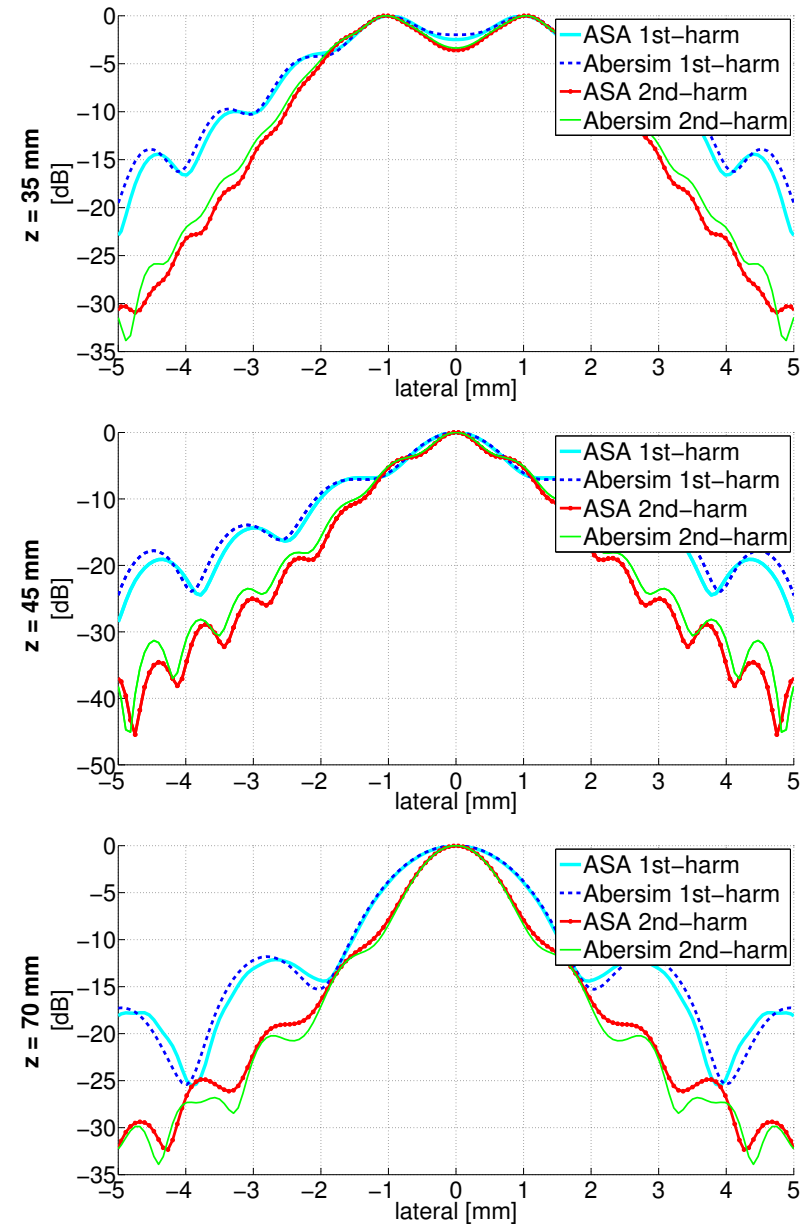

Fig. 4: Comparisons at three different depths $(z=35 \mathrm{~mm}, z=$ $45 \mathrm{~mm}, z=70 \mathrm{~mm}$ from the transducer surface) between FieldII-based ASA and Abersim for both the fundamental and second harmonic components

\begin{tabular}{l|ccccc} 
& $\mathrm{nz}=2$ & $\mathrm{nz}=4$ & $\mathrm{nz}=6$ & $\mathrm{nz}=8$ & $\mathrm{nz}=10$ \\
\hline AS-Fd-1 & $21.2 \%$ & $7.68 \%$ & $1.89 \%$ & $3.31 \%$ & $2.66 \%$ \\
AS-Ab-1 & $24.2 \%$ & $8.86 \%$ & $3.82 \%$ & $4.82 \%$ & $4.28 \%$ \\
AS-Ab-2 & $7.73 \%$ & $0.93 \%$ & $0.86 \%$ & $0.80 \%$ & $0.91 \%$ \\
CT & $85 \mathrm{~s}$ & $0.5 \mathrm{~h}$ & $2 \mathrm{~h}$ & $8 \mathrm{~h}$ & $20 \mathrm{~h}$ \\
\hline \multicolumn{2}{l}{ CT: Computation Time } & \multicolumn{5}{c}{ nz: zero-padding number }
\end{tabular}

TABLE III: RMS errors and computation time of Field-II-based ASA compared with Abersim and Field II using different zero-paddings

splitted into 50 independent jobs and run on 50 PCs with 4 cores Intel(R) Xeon ${ }^{\mathrm{TM}} 3.06 \mathrm{GHz}$ CPU and $2 \mathrm{~GB}$ memory, and the computation time of ASA is only 15 minutes by Matlab 6.5.0 under the Mandrake Linux 10.0 operating system when the zero-padding number is 6 . The FWHMs of the mainlobe for different zero-padding numbers of ASA are shown in Table IV compared with the results from Abersim and Field II. The good agreement is also obtained between different methods.

\section{CONCLUSION}

Field-II-based ASA for simulating both fundamental and second harmonic components gave very similar results compared to Abersim at arbitrary depth. The combination of Field II

\begin{tabular}{c|cc|cc|c} 
& \multicolumn{2}{|c|}{ Field-II-based ASA } & \multicolumn{2}{c|}{ Abersim } & Field II \\
& 1st-harm & 2nd-harm & 1st-harm & 2nd-harm & 1st-harm \\
\hline $\mathrm{nz}=6$ & $2.00 \mathrm{~mm}$ & $1.20 \mathrm{~mm}$ & & & \\
$\mathrm{nz}=8$ & $2.02 \mathrm{~mm}$ & $1.20 \mathrm{~mm}$ & $2.07 \mathrm{~mm}$ & $1.24 \mathrm{~mm}$ & $2.04 \mathrm{~mm}$ \\
$\mathrm{nz}=10$ & $2.02 \mathrm{~mm}$ & $1.21 \mathrm{~mm}$ & & &
\end{tabular}

TABLE IV: FWHMs of the mainlobe for different zero-paddings of Field-II-based ASA compared with Abersim and Field II

and ASA makes it possible to simulate non-linear ultrasound imaging for any kind of transducer, focusing, apodization and attenuation for a monochromatic field. Using Field-II-based ASA planes at any depth can be calculated based on the emission source and distance from it and only one calculation step is required. Using Abersim based on the OSM, a number of calculations are needed, if the simulated planes are far from the source. Therefore, the monochromatic calculation of the pressure for a specified point in ultrasound fields using ASA is faster than that of using Abersim.

\section{ACKNOWLEDGMENT}

This work was supported by grant 26-04-0024 by the Danish Science Foundation and BK Medical Aps, Herlev, Denmark.

\section{REFERENCES}

[1] J. A. Jensen and N. B. Svendsen. Calculation of pressure fields from arbitrarily shaped, apodized, and excited ultrasound transducers. IEEE Trans. Ultrason., Ferroelec., Freq. Contr., 39(2):262-267, 1992.

[2] J. A. Jensen. Field: A program for simulating ultrasound systems. In Med. Biol. Eng. Comp., volume 34, pages 351-353, 1996.

[3] J. A. Jensen, P. D. Fox, J. Wilhjelm, and L. K. Taylor. Simulation of non-linear ultrasound fields. In Proc. IEEE Ultrason. Symp., volume 2, pages 1733-1736, 2002.

[4] T. Varslot and G. Taraldsen. Computer simulation of forward wave propagation in soft tissue. IEEE Trans. Ultrason., Ferroelec., Freq. Contr., 52(9):1473-1482, 2005.

[5] T. Varslot and S. E. Måsøy. Forward propagation of acoustic pressure pulses in 3D soft biological tissue, Modeling, Identification and Control. IEEE Trans. Ultrason., Ferroelec., Freq. Contr., 27(3):181-200, 2006.

[6] M. E. Frijlink, H. Kaupang, T. Varslot, and S.-E. Måsøy. Abersim: a simulation program for $3 \mathrm{D}$ nonlinear acoustic wave propagation for arbitrary pulses and arbitrary transducer geometries. In Proc. IEEE Ultrason. Symp., pages 1282-1285, 2008.

[7] H. Kaupang. Abersim 2.x Reference manual with tutorials. Norwegian University of Science and Technology, 2008.

[8] M. F. Hamilton and D. T. Blackstock. Nonlinear Acoustics: Theory and Application. Academic Press, 1998.

[9] B. J. Landsberger and M. F. Hamilton. Second-hamonic generation in sound beams reflected from, and transmitted through, immersed elastic solids. J. Acoust. Soc. Am., 109(2):488-500, 2001.

[10] Y. Xiang and M. F. Hamilton. Angular spectrum decomposition analysis of second harmonic ultrasound propagation and its relation to tissue harmonic imaging. In Ultrasonic and Advanced Methods for Nondestructive Testing and Material Characterization, volume UmassDNDT 2006, pages 11-24, 2006.

[11] P. J. Westervelt. Parametric acoustic array. J. Acoust. Soc. Am., 35(4):535-537, 1963.

[12] J. N. Tjotta and S. T. Tjotta. Interaction of sound waves. Part I: Basic equations and plane waves. J. Acoust. Soc. Am., 82(4):1425-1428, 1987.

[13] Y. Xiang. Statistical Model of Beam Distortion by Tissue Inhomogeneities in Tissue Harmonic Imaging. PhD thesis, The University of Texas at Austin, December 2004.

[14] Y. Du and J. A. Jensen. Feasibility of non-linear simulation for Field II using an angular spectrum approach. In Proc. IEEE Ultrason. Symp., pages 1314-1317, 2008.

[15] L. K. Taylor, M. Schlaikjer, and J. A. Jensen. Modeling of higher harmonics formation in medical ultrasound systems. In Med. Imag. V Symp., volume 4687, pages 59-67, 2002.

[16] J. A. Jensen. Users' guide for the Field II program. Technical University of Denmark, August 2001. 